\title{
Pigment-Dispersing Hormone Shifts the Phase of the Circadian Pacemaker of the Cockroach Leucophaea maderae
}

\author{
Bernhard Petri and Monika Stengl \\ Institut für Zoologie/Biologie I, Universität Regensburg, 93040 Regensburg, Germany
}

An antiserum against the crustacean neuropeptide pigmentdispersing hormone stains a small set of neurons in the optic lobes of several hemimetabolous and holometabolous insects. These cells, the primary branches of which in the optic lobe lie in the accessory medulla, fulfill several criteria predicted for neurons of the circadian clock. For example, in fruit flies they express timeless and period, which are two molecular components of the circadian pacemaker.

To test whether pigment-dispersing hormone fulfills a circadian function in the cockroach Leucophaea maderae, $150 \mathrm{fmol}$ of synthetic peptide was injected into the vicinity of the accessory medulla. This resulted in a stable phase-dependent reset- ting of the phase of the circadian locomotor activity rhythm, which depended on the amount of pigment-dispersing hormone injected. The resulting phase-response curve differs from that obtained with light pulses, suggesting that pigmentdispersing hormone-immunoreactive neurons are not part of the visual input pathway to the pacemaker but an integral part of it and/or part of a nonphotic input into the clock. A possible role of these neurons in coupling the bilaterally paired circadian pacemakers is discussed.

Key words: pigment-dispersing hormone; neuropeptides; circadian rhythms; phase shifts; pacemaker; insects
The temporal structure of physiological and behavioral states is probably organized by internal clocks in all organisms. In the circadian system, this clock comprises a circadian pacemaker, which generates an endogenous rhythm of about $24 \mathrm{hr}$. The circadian system also includes an entrainment pathway for the synchronization with zeitgebers and output pathways to effector organs.

Lesion studies have localized bilaterally paired circadian pacemakers in the optic lobes, ventrally between medulla and lobula of orthopteromorph insects such as the cockroach Leucophaea maderae (Nishiitsutsuji-Uwo and Pittendrigh 1968a,b; Roberts 1974; Sokolove 1975; Wills et al. 1985; for review, see Page, 1985; Chiba and Tomioka, 1987). These mutually coupled pacemakers control the phase and the period of the circadian locomotor activity rhythm as well as circadian changes in the sensitivity of the compound eyes (Page et al., 1977; Page, 1978, 1982, 1983a; Wills et al., 1985; Colwell and Page, 1989, 1990). Both pacemakers can be entrained by photoreceptors in or near the compound eyes (Roberts, 1965; Nishiitsutsuji-Uwo and Pittendrigh, 1968a; Page et al., 1977; Page, 1983a,b) and receive coupling input from the contralateral pacemaker (Page, 1981). However, the neuronal substrate of the circadian pacemaker and its input and output pathways remain elusive.

A small discrete neuropil was discovered in the region where the lesion studies had located the circadian clock in hemimetabolous insects (Homberg et al., 1991). This neuropil, which was termed the accessory medulla (AMe), is innervated by a small group of neurons with adjacent somata, which are immunoreac-

Received Jan. 6, 1997; revised March 10, 1997; accepted March 19, 1997.

This research was supported by Deutsche Forschungsgemeinschaft Grants Ste 531/1-1, Ho 950/9-1, and Ste 531/7-1. We are grateful to Dr. K. R. Rao for the generous supply of PDH peptides and Dr. T. Roenneberg for the Chrono II software. Furthermore, we thank G. Stoeckl, R. Zintl, and S. Gries for technical support and Drs. Uwe Homberg and J. Kien for critical reading of this manuscript.

Correspondence should be addressed to Dr. Monika Stengl at the above address Copyright (C) 1997 Society for Neuroscience $0270-6474 / 97 / 174087-07 \$ 05.00 / 0$ tive with an antiserum against the neuropeptide pigmentdispersing hormone (PDH) (Homberg et al., 1991). These PDHimmunoreactive neurons fulfill morphological criteria predicted for elements of the circadian system such as the specific location of their somata and primary branching areas in the optic lobes and arborizations in several areas of the midbrain (Sokolove, 1975; Page, 1985; Homberg et al., 1991; Nässel et al., 1991; Stengl and Homberg, 1994). Mass-staining techniques such as immunocytochemistry, however, cannot discern the branching patterns of individual $\mathrm{PDH}$-immunoreactive neurons. Intracellular recordings and Lucifer yellow staining of single neurons suggest that individual PDH-immunoreactive neurons differ in their morphologies and functions (Würden and Homberg, 1994; Lösel and Homberg, 1996). Recent experiments in cockroaches, crickets, and flies suggest the following functions for different individual PDH-immunoreactive neurons: (1) they could be the neurons of the light entrainment pathway (Homberg et al., 1991; Würden and Homberg, 1994; Lösel and Homberg, 1996); (2) they could be output pathways to the effectors of the clock (Helfrich-Förster and Homberg, 1993; Stengl and Homberg, 1994; Pyza and Meinertzhagen, 1995, 1996; Stengl, 1995; Meinertzhagen and Pyza, 1996; Helfrich-Förster, 1997); (3) they may couple the bilaterally paired optic lobe pacemakers (Stengl and Homberg, 1994); and (4) they may themselves be an integral part of the pacemaker (Helfrich-Förster, 1995).

This study examines the functions of $\mathrm{PDH}$, the presumptive neurotransmitter and neuromodulator of PDH-immunoreactive neurons, via injection of a peptide into the vicinity of the AMe. We show that PDH specifically shifts the phase of the pacemaker that drives the circadian wheel-running activity rhythm of $L$. maderae. Our results cannot distinguish between the functions of different individual PDH-immunoreactive neurons, but they suggest that at least some of these neurons are an integral part of the pacemaker and/or part of a nonphotic input. 

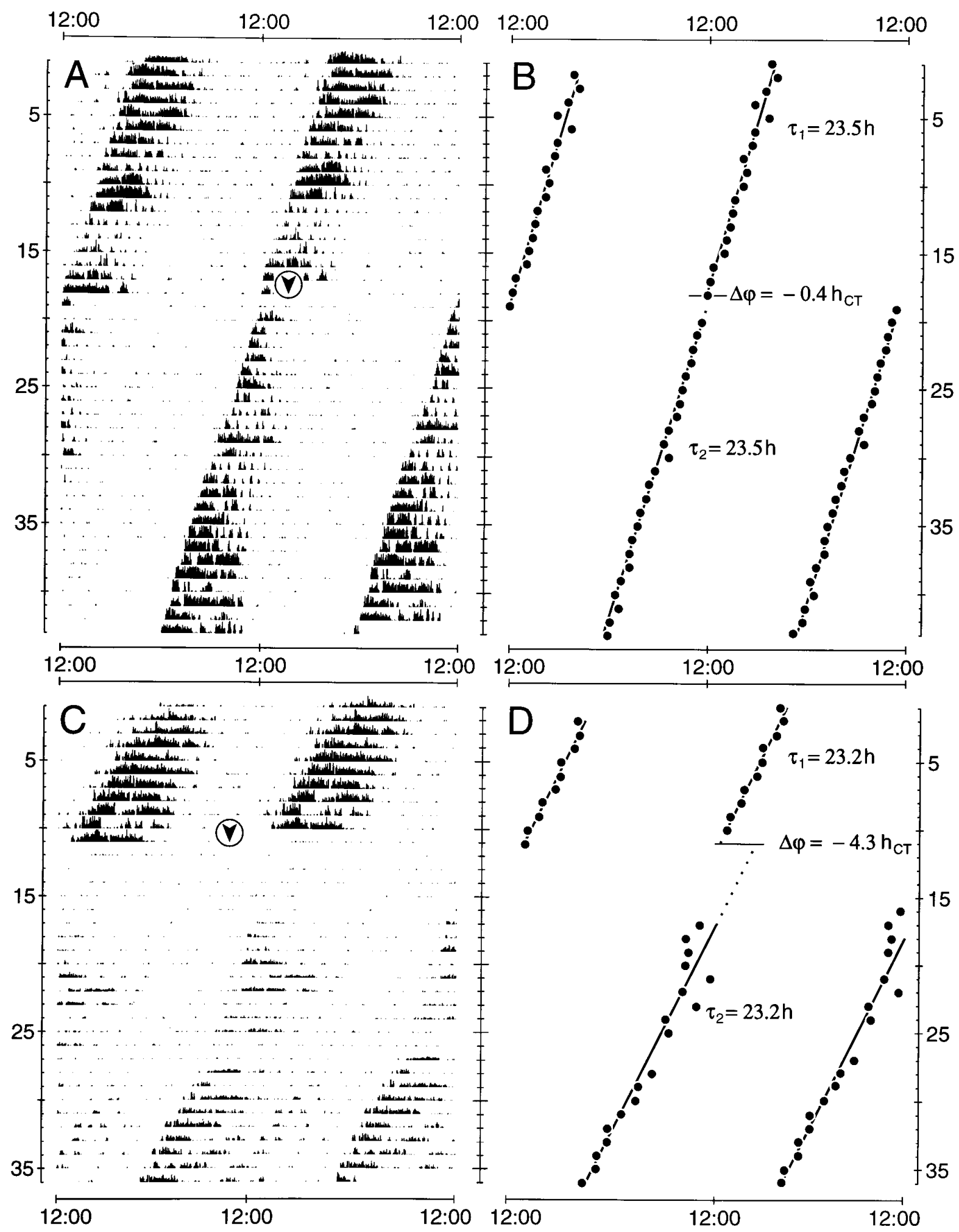

Figure 1. Records of circadian wheel-running activity and plots of activity onsets from cockroaches kept in constant darkness. $A$, $B$, After an injection of $46 \mathrm{fmol}$ of synthetic PDH in $0.5 \mathrm{nl}$ of saline at CT 13:00 of day 19 (arrowhead), regression analysis through consecutive activity onsets $(B)$ reveals a resulting phase delay of -0.4 circadian $\mathrm{hr}\left(h_{C T}\right)$ after the injection. $C$, $D$, Injection of $46 \mathrm{fmol}$ of synthetic PDH in 0.5 nl of saline at CT 9:00 of day 12 (arrowhead) induces a phase delay of -4.3 circadian $\mathrm{hr}\left(h_{C T}\right)$. $A-D$, In both experiments, no effect on the period $\tau$ of the activity rhythm is apparent. $x$-axis, Time of the day; $y$-axis, days. 


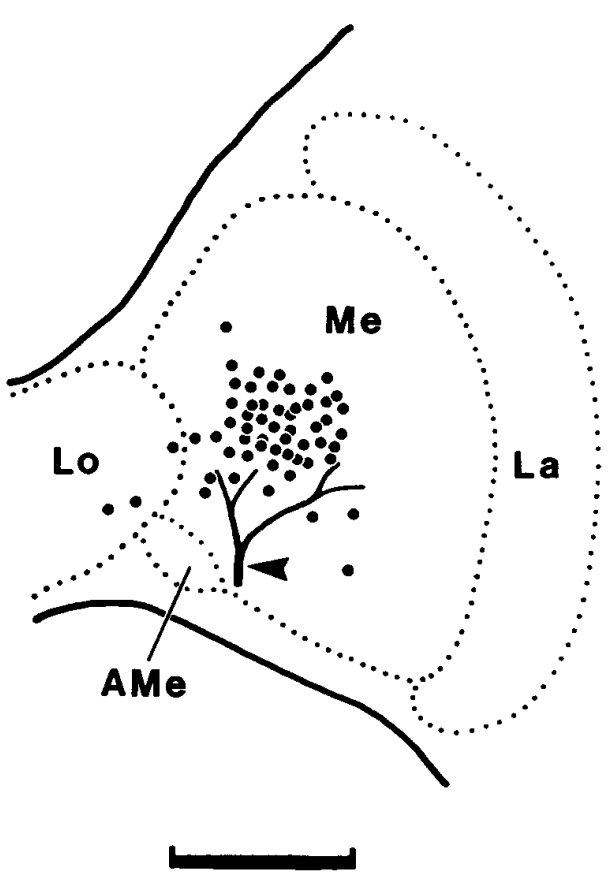

Figure 2. Schematic drawing of the different PDH injection sites. PDH was pressure injected via glass microcapillaries into the vicinity of the AMe. Each dot represents the location of one injection, as assessed by visual control during the experiment $(n=57)$. A characteristic trachea at the surface of the optic lobe (arrowhead) was used as a landmark. Me, Medulla; $L a$, lamina; $L o$, lobula. Scale bar, $200 \mu \mathrm{m}$.

\section{MATERIALS AND METHODS}

Beavioral assays and data analysis. L. maderae cockroaches were reared in laboratory colonies at $30^{\circ} \mathrm{C}, 30 \%$ humidity, and light/dark cycles of $12: 12$ $\mathrm{hr}$ (with lights on from 6 A.M. to 6 P.M.). Only males were chosen for the experiments $(n=141)$, because they expressed a more robust circadian locomotor activity rhythm than females. Behavioral analysis was performed in constant darkness (DD), at constant temperature $\left(28 \pm 0.5^{\circ} \mathrm{C}\right)$, and constant humidity (70\%). Experimental animals were continuously provided with food (dry rat pellets) and water. Locomotor activity was recorded in running wheels (modified from running wheels provided by Dr. Wolfgang Engelmann, University of Tübingen, Tübingen, Germany; described by Wiedenmann, 1977) equipped with a magnetic reed switch. One revolution of the running wheel resulted in one impulse. The impulses were continuously counted by a microcomputer in 1 min intervals and condensed and processed by custom made PC-compatible software (developed by H. Fink, University of Konstanz, in collaboration with Drs. M. Stengl and F. Wollnik). This software allows an on-line visualization of the raw data in activity histograms as well as $\chi^{2}$ periodogram analysis. The data were plotted in double plot activity histograms. The heights of the bars represent the number of revolutions per $5 \mathrm{~min}$; they were cut off at $30 \mathrm{rpm}$.

The free-running period, $\tau$, and the induced phase shifts were estimated by converting the raw data into ASCII format. They were then merged into 20 min intervals and analyzed with CHRONO II software (generously provided by Dr. Till Roenneberg; see Roenneberg and Morse, 1993) on an Apple Macintosh computer (data were obtained from 104 animals). The remaining 37 animals were excluded from further analysis because they showed little activity after the injection or died within 1 week after the operation.

The free-running period $\tau$ before and after the injection was calculated by linear regression through daily activity onsets and by $\chi^{2}$ periodogram analysis (Sokolove and Bushell, 1978; Enright, 1965). Changes in free-running period $\tau\left(\Delta \tau=\tau_{\text {after }}-\tau_{\text {before }}\right)$ were calculated with periods estimated by regression through activity onsets. Phase shifts $(\Delta \varphi)$ were estimated by measuring the time difference between the regression lines before and after the injection extrapolated to the day of the treatment (Fig. 1). These phase shifts were then normalized with respect to the free-running period $\tau$ before the treatment. Phase delays are plotted as negative values, and phase advances are plotted as positive values. Daily activity onsets were determined by CHRONO II (see Roenneberg and Morse, 1993). The time on the $x$-axis was indicated as circadian time (CT), with $\mathrm{CT} 12: 00=$ activity onset = beginning of the subjective night.

The microinjection data were merged into $2 \mathrm{hr}$ intervals, and the means, SEM, and SD were calculated. Changes in phase and period in a given interval were considered significantly different from 0 when the calculated $95 \%$ confidence interval (CI) of that interval did not contain the value 0 (Sachs, 1969). The phase-response curves were analyzed by one-way ANOVA with Scheffé's multiple range test. Significance in all cases was defined as $p<0.05$. Statistical analyses were performed with Superior Performing Software Systems (SPSS Inc.) on a personal computer.

Operation and injection. All manipulations were performed in dim red light at room temperature $\left(25^{\circ} \mathrm{C}\right)$ with a Microinjector (model 5242;

Table 1. Phase shifts (mean \pm SEM in circadian hours) resulting from PDH and control injections at different times of the circadian cycle (circadian time)

\begin{tabular}{llccr} 
Injection & Circadian time & Phase shift & 95\% CI & $n$ \\
\hline PDH & $00: 00-02: 00$ & $0.8 \pm 0.2^{a}$ & -0.3 to 1.8 & 3 \\
& $02: 00-04: 00$ & $0.3 \pm 0.1^{a}$ & -0.1 to 0.6 & 5 \\
& $04: 00-06: 00$ & $-0.2 \pm 0.4^{a}$ & -2.1 to 1.7 & 3 \\
& $06: 00-08: 00$ & $-1.1 \pm 0.3^{a}$ & -2 to $-0.2^{b}$ & 5 \\
& $08: 00-10: 00$ & $-3.2 \pm 0.4^{c, d}$ & -4 to $-2.2^{b}$ & 11 \\
& $10: 00-12: 00$ & $-2.2 \pm 0.5^{d}$ & -3.4 to $-1^{b}$ & 8 \\
& $12: 00-14: 00$ & $-1.4 \pm 0.2^{a}$ & -1.7 to $-0.9^{b}$ & 2 \\
& $14: 00-16: 00$ & $-0.9 \pm 0.4$ & -6 to 4.2 & 5 \\
& $16: 00-18: 00$ & $0 \pm 0.3^{a}$ & -0.8 to 0.8 & 5 \\
& $18: 00-20: 00$ & $0.6 \pm 0.5^{a}$ & -0.8 to 2.1 & 4 \\
Control & $20: 00-22: 00$ & $0.7 \pm 0.4^{a}$ & -0.7 to 2 & 3 \\
& $08: 00-10: 00$ & $0 \pm 0.2$ & -1 to 1 & 5 \\
& $10: 00-12: 00$ & $-0.3 \pm 0.1$ & -0.6 to 0.1 & 35
\end{tabular}

${ }^{a}$ PDH-dependent phase shifts significantly different from that at circadian time 08:00-10:00.

${ }^{b}$ Statistically significant phase shifts after PDH injections, as judged by the 95\% CI (see Materials and Methods).

${ }^{c}$ Phase shifts additionally significantly different from PDH-dependent phase shifts at other circadian times $\left({ }^{a}\right)(p<0.05$, Scheffé's multiple range test).

${ }^{d}$ PDH-dependent phase shifts that are significantly different from control injections $(p<0.05$ Scheffé's multiple range test). 


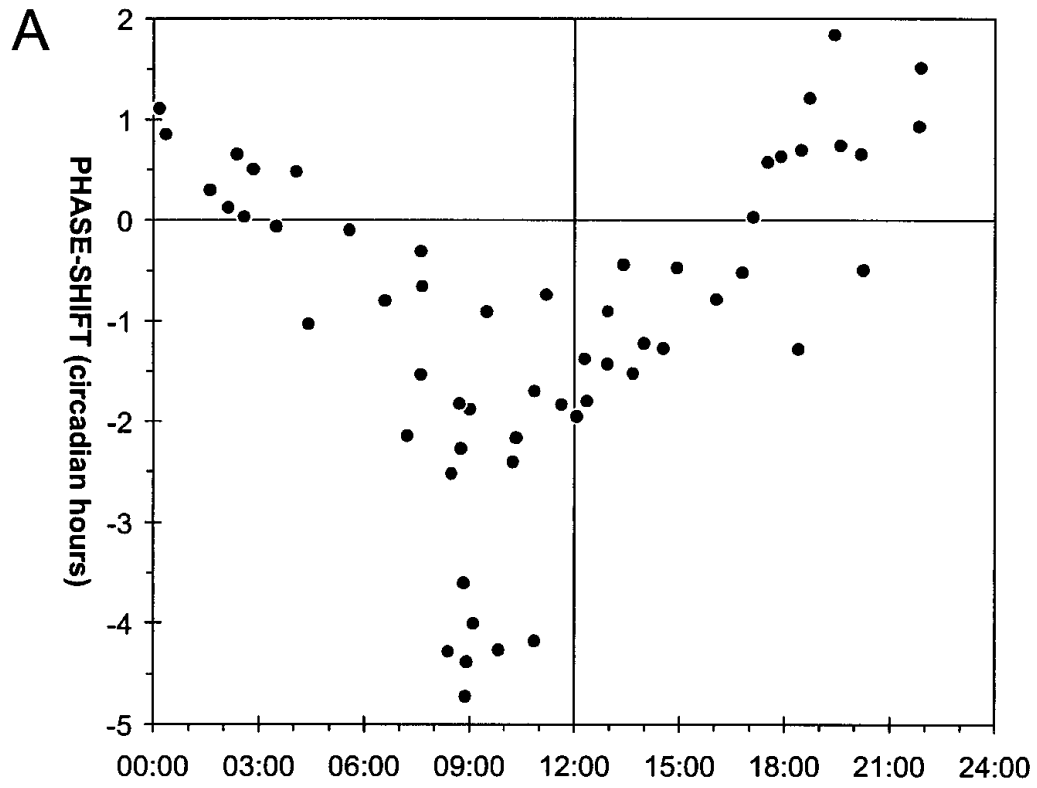

Figure 3. Scatter plot of PDH-dependent phase shifts at different times in the circadian cycle. $A$, The PDH injections $(150 \pm 60 \mathrm{fmol}$ in $1.5 \pm 0.6 \mathrm{nl}$ of saline, mean $\pm \mathrm{SD} ; n=57)$ cause maximal phase delays during the late subjective day $(-4.7 \mathrm{hr}$ at CT 8:50) and maximal phase advances during the late subjective night and early subjective day $(1.8 \mathrm{hr}$ at CT 18:30). The phase advances were not statistically significant. $B$, Independent of the time of day, control injections $(0.5-2 \mathrm{nl}$ of saline; $n=35$ ) caused only small phase delays and phase advances, which were not statistically significant. Each point represents the phase shift (in circadian hours) resulting from a single injection. Phase advances are always shown as positive values, and phase delays are shown as negative values.

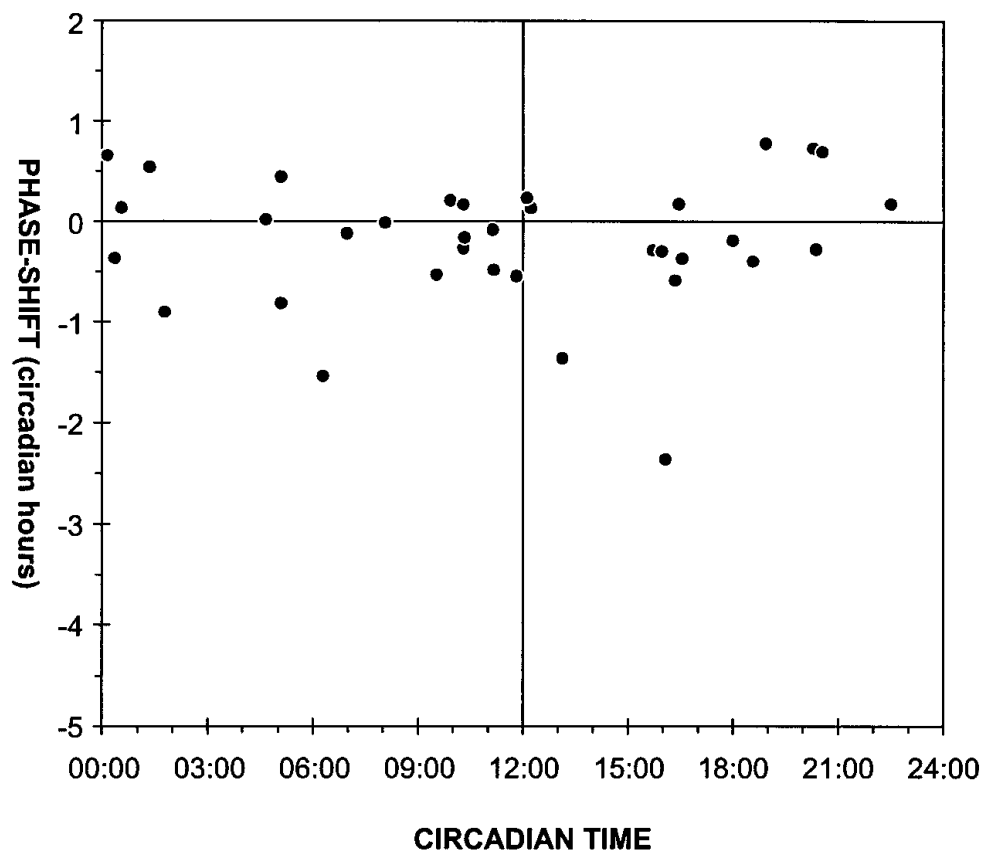

Eppendorf, Hamburg, Germany). Experimental animals were removed from the running wheels and mounted in metal tubes at different times during the circadian cycle. After anesthetizing them with $\mathrm{CO}_{2}$, a small window was cut into the head capsule, and the optic lobe was revealed by moving the trachea, ocellus, and fat body carefully to the side. The neurolemma of the optic lobe was penetrated with a borosilicate glass capillary (Clark, Pangbourne Reading, England), and the neuropeptides were pressure injected under visual control into one medulla, dorsally and distally to the AMe (Fig. 2). The region of the AMe can be localized accurately using external landmarks such as the trachea shown in Figure 2. After the injection the removed piece of cuticle was waxed back, and the animal was returned to the running wheel. Each treatment lasted $10-15 \mathrm{~min}$. All injections targeted only one optic lobe, whereas the other lobe was left intact. It was known from earlier studies that both bilaterally symmetric pacemakers are tightly coupled in cockroaches (Page, 1981, 1985). This means that any disturbance of one pacemaker will be transferred to the other one and will result in a phase shift of the locomotor activity rhythm (which, therefore, is controlled by both coupled pacemak- ers together). All experimental animals were kept under constant conditions (DD) before and after the injection.

Because PDH from L. maderae has not been sequenced yet, neuropeptide injections consisted of $10^{-4}-10^{-12} \mathrm{M}$ synthetic $\mathrm{Arg}^{13}$-Acheta domestica PDH (NSEIINSLLGLPRVLNDA-amide; generously provided by Dr. K. R. Rao, University of West Florida, Pensacola, FL) in $10 \%$ aqueous blue food dye (McCormick, Baltimore, MD), which made the exact site of the injection visible without the need for further neuroanatomical processing of the brain. The purity of the synthetic peptide was examined by HPLC and by quantitative amino acid analysis (performed by R. Rao and associates). A peptide concentration of $10^{-4} \mathrm{M}$ was used for obtaining the phase-response curve. Concentrations of $10^{-8}$ and $10^{-12} \mathrm{M}$ PDH were also tested to plot the dose-response curve. Each micropipette was calibrated by estimating the injected volume. Droplets were injected into mineral oil before and after the injection to control for changes in tip diameter during penetration of the neurolemma. The injected volume for all 104 injections ranged from 0.5 to $2 \mathrm{nl}$ with a mean 


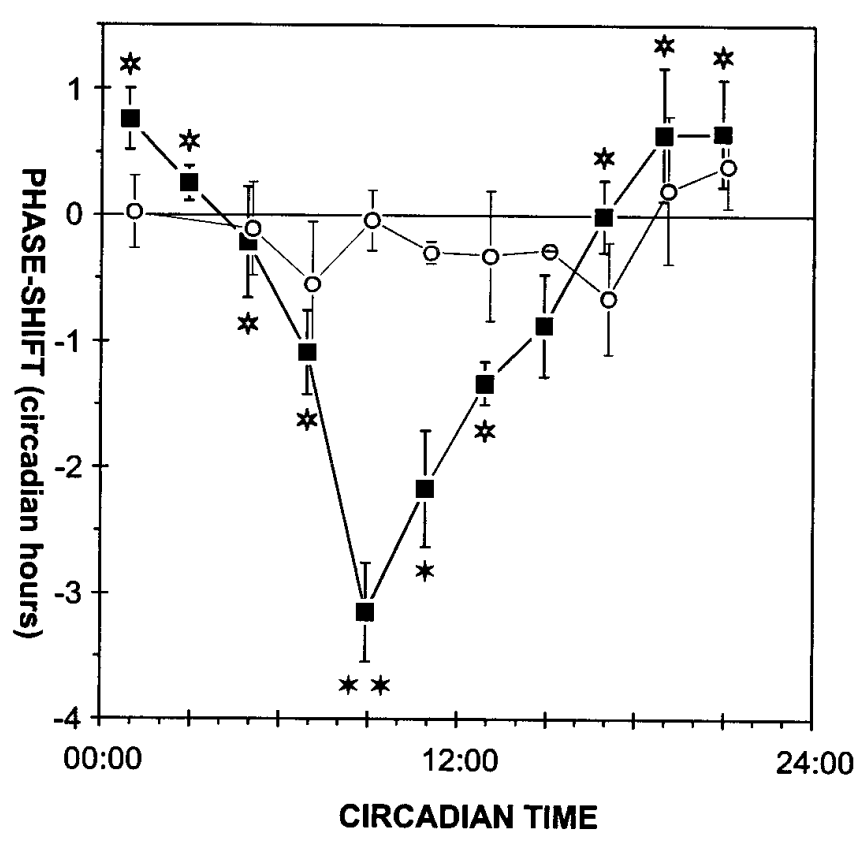

Figure 4. Phase-response curves obtained with PDH or control injections. Data from Figure 3 were merged into $2 \mathrm{hr}$ bins. The PDHdependent phase shifts (closed squares) and phase shifts after control injections (open circles) are plotted (mean \pm SEM) in the middle of each $2 \mathrm{hr}$ bin. All filled stars indicate PDH-dependent phase delays that are significantly different from phase shifts following control injections. Furthermore, PDH-dependent phase delays at CT 8:00-10:00 (double star) are significantly different from $\mathrm{PDH}$ injections at other circadian times (open stars) $(p<0.05$, Scheffé's multiple range test).

dose of $1.5 \pm 0.6(\mathrm{SD}) \mathrm{nl}$. Control injections consisted of $10 \%$ aqueous blue food dye without PDH.

\section{RESULTS}

\section{Effects of PDH injections on the phase of the circadian locomotor activity rhythm}

Control injections of blue food dye caused no significant phase change in circadian locomotor activity or rhythm, regardless of when in the circadian cycle it was injected (Table 1). Microinjections of synthetic PDH $(n=57)$ into the medulla resulted in time-dependent phase shifts of the circadian activity rhythm of $L$. maderae (Figs. 1, 2, Table 1). Maximal phase delays $(-4.7 \mathrm{hr})$ occurred when PDH was given late in the subjective day (CT $8: 50)$, and maximal phase advances $(1.8 \mathrm{hr}$ ) occurred with injections late in the subjective night (CT 19:30) (Fig. 3A). Examination of the $95 \%$ confidence intervals (95\% CIs) for the phase shifts in different $2 \mathrm{hr}$ bins (see Materials and Methods) indicates that significant peptide-dependent phase shifts occurred at CT 6:00-14:00 (Table $1,{ }^{b}$ ). The shifts during the rest of the cycle, including the phase advances, were not significant.

This phase dependency was statistically significant $(p<$ 0.00005, one-way ANOVA), because PDH-dependent phase delays at CT 8:00-10:00 were significantly different from $\mathrm{PDH}$ dependent phase shifts during other times of the circadian cycle (Table $1,{ }^{c}$, Fig. 4). In addition, delays induced by PDH injections at CT 8:00-10:00 and 10:00-12:00 were significantly different from the effects of the control injections at the same circadian times (Table 1, ${ }^{c, d}$, Fig. 4). Effects of control injections in different intervals were not significantly different from each other $(p=0.7$,

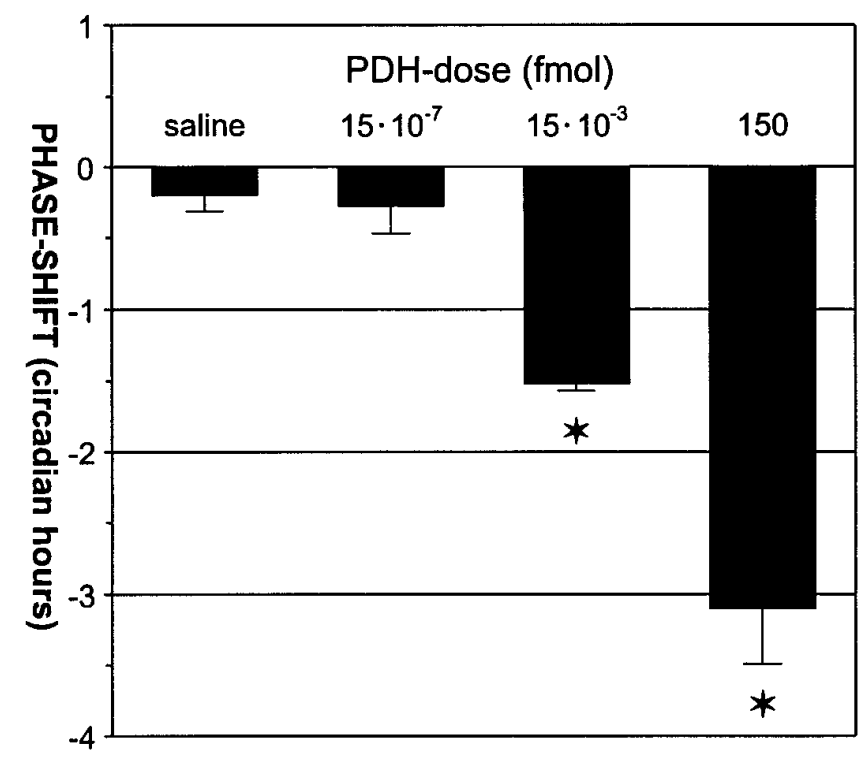

Figure 5. Dose dependency of PDH-induced phase shifts between CT 8:00 and CT 10:00. Each bar represents the mean phase shift (in circadian hours \pm SEM) resulting from injections of saline $(n=35), 15 \times 10^{-7} \mathrm{PDH}(n=8)$, $15 \times 10^{-3} \mathrm{PDH}(n=4)$, and $150 \mathrm{fmol}$ of PDH $(n=11)$ in $1.5 \mathrm{nl}$ of saline. Stars indicate PDH doses that induced phase shifts significantly different from control (saline) injections ( $p<0.05$, Scheffé's multiple range test).

one-way ANOVA), and in no case were they significantly different from $0 \mathrm{hr}$ (Table 1).

\section{Effects of PDH injections on the period of the circadian locomotor activity rhythm}

No significant changes in the free-running period (Fig. 1) of individual experimental cockroaches after the injection of either saline or PDH were found ( $p=0.6$, one-way ANOVA). The effects observed were always small and included both lengthening (by maximally $0.3 \mathrm{hr}$ ) and shortening (by maximally $-0.4 \mathrm{hr}$ ) and were independent of the time of injection in the circadian cycle. On average, the mean period $(23.4 \pm 0.2 \mathrm{hr}$, mean $\pm \mathrm{SD} ; n=92)$ was not altered either by PDH or by control injections $(0 \pm 0.2 \mathrm{hr}$, mean $\pm \mathrm{SD} ; 95 \% \mathrm{CI},-0.1$ to $0.1 \mathrm{hr} ; n=92)$. In four experiments (as in Fig. 1C) the period up to 10 days after the injection seemed longer than the average period. However, because of little overall activity after the operation, we were not able to confirm this observation by periodogram analysis.

\section{Dose dependency of PDH-dependent phase shifts}

Microinjection of synthetic PDH into the optic lobes of cockroaches at CT 8:00-10:00 caused phase delays in circadian wheelrunning activity that depended on the dose of PDH injected (Fig. 5 ). The phase delays decreased with decreasing amounts of injected PDH. Significant phase delays (Fig. 5, stars) were caused by injection of $150 \mathrm{fmol} \mathrm{PDH}(-3.1 \pm 0.4 \mathrm{hr}$; $95 \% \mathrm{CI},-4$ to $-2.3 \mathrm{hr}$; $n=11)$ and $15 \times 10^{-3}$ fmol PDH $(-1.5 \pm 0.1 \mathrm{hr} ; 95 \% \mathrm{CI},-1.7$ to $-1.4 \mathrm{hr} ; n=4)$. However, injection of $15 \times 10^{-7} \mathrm{fmol} \mathrm{PDH}$ caused phase shifts $(-0.3 \pm 0.2 \mathrm{hr} ; 95 \% \mathrm{CI},-0.7$ to $0.2 \mathrm{hr} ; n=$ $8)$ that were indistinguishable from control injections $(-0.2 \pm 0.1$ hr; $95 \% \mathrm{CI},-0.4$ to $0.1 \mathrm{hr} ; n=35$ ) ( $p<0.05$, one-way ANOVA, Scheffé's multiple range test).

\section{DISCUSSION}

Microinjections of the neuropeptide PDH into the vicinity of the $\mathrm{AMe}$, the predicted pacemaker region, resulted in statistically 


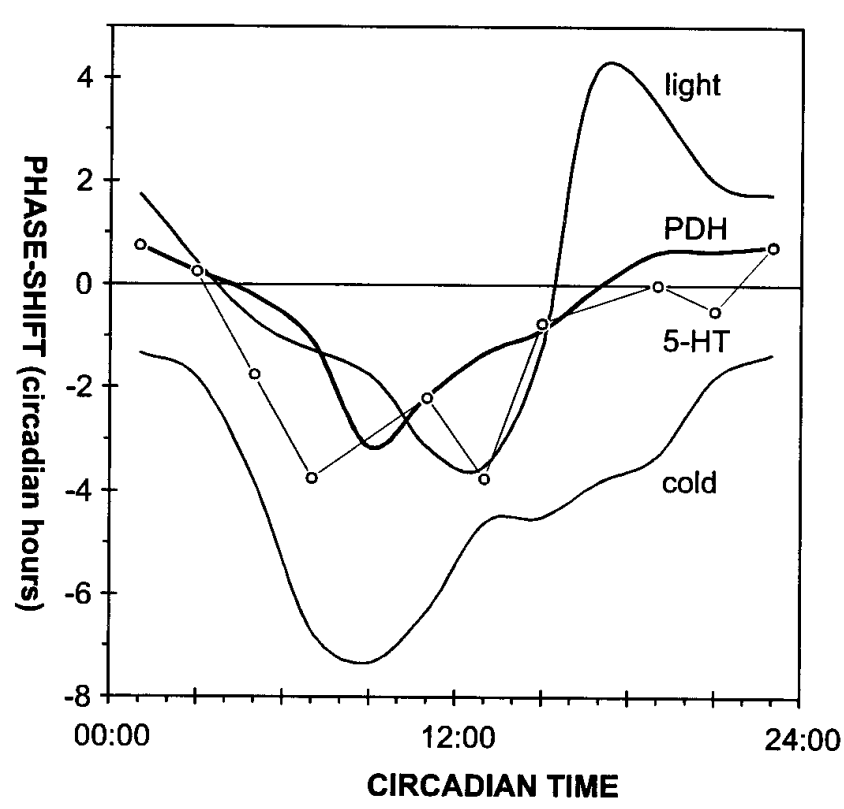

Figure 6. Phase-response curves for different phase-shifting signals in $L$. maderae. The phase-response curve for PDH is similar in shape to the phase-response curve obtained for low temperature pulses (cold) (Wiedenmann, 1971) and serotonin infusions (5-HT) (Page, 1987), but it is different from that obtained with light pulses (light) (Page and Barret, 1989).

significant phase delays of the circadian wheel-running activity rhythm during the late subjective day. Therefore, the neuropeptide PDH seems to be a component of the circadian system of the cockroach L. maderae.

Our results, together with immunocytochemical studies (Homberg et al., 1991; Stengl and Homberg, 1994; Petri et al., 1995; Reischig and Stengl, 1996) indicate that PDH-immunoreactive processes in the AMe mediate nonphotic inputs into the clock and/or are an integral part of the pacemaking neuronal network. Further neuroanatomical studies on the light and electronmicroscopic level have to decipher the individual branching patterns of individual $\mathrm{PDH}$-immunoreactive neurons with arborizations in the AMe that seem to fulfill these functions.

\section{Dose dependency of PDH-dependent phase shifts}

The phase-dependent phase shifts of the locomotor activity rhythm in the late subjective day are specifically dependent on PDH, because they are dose dependent (Figs. 4, 5) and significantly different from control injections. Furthermore, preliminary injections of another peptide (Mas-allatotropin) indicate that different peptides cause completely different phase-response curves (B. Petri and M. Stengl, unpublished observations). The threshold for PDH-dependent phase shifts lies between 0.015 and $1.5 \times 10^{-6} \mathrm{fmol}$ of PDH. This seems to be a physiological dose level, because single brains of orthopteromorph insects contain 1.2-50 pmol of PDH, with about $90 \%$ of the PDH immunoreactivity concentrated in the optic lobes (Rao and Riehm, 1993; S. Würden, personal communication).

So far, PDH-related peptides have not been sequenced from $L$. maderae, but comparison of the primary structure of PDH-related peptides reveals a highly conserved sequence between crustaceans and different insects (Rao and Riehm, 1993). The PDH, which was biologically active in our experiments ( $\mathrm{Arg}^{13}$-Acheta domestica $\mathrm{PDH}$ ), differs only at two amino acid residues (positions 4 and
13) from the PDH of the cockroach Periplaneta americana (Rao and Riehm, 1993). This suggests that a peptide closely related to the $A$. domestica $\mathrm{PDH}$ is released by the $\mathrm{PDH}$-immunoreactive neurons of L. maderae.

\section{A possible role of $\mathrm{PDH}$-immunoreactive neurons as pacemaker neurons and/or input pathways to the pacemaker}

Injection of PDH maximally delays the clock at times of the day when light has only weak effects, and conversely, $\mathrm{PDH}$ has no effects at times when light advances the clock (Fig. 6) (Page, 1987; Page and Barret, 1989). Therefore, it is unlikely that PDHimmunoreactive neurons serve as photic input to the pacemaker.

The phase-response curve obtained after PDH injections is comparable to the nonphotic phase-response curves obtained with serotonin or temperature pulses (Fig. 6) (Wiedenmann, 1977; Page, 1987). All three phase-response curves are monophasic with the maximal delay region occurring at different times of the late subjective day (Fig. 6) (Wiedenmann, 1977; Page, 1987). The phase delay amplitude resulting from $\mathrm{PDH}$ injections is similar to that caused by serotonin pulses (Fig. 6) (Page, 1987), but the serotonin curve seems to have a broader peak. This is possibly attributable to different experimental methods. Page (1987) infused doses of serotonin 7 log units higher than the PDH doses used here into the hemolymph during periods of $6 \mathrm{hr}$. This may have resulted in a broadening of the delay peak. Because an excessive number of indistinguishable 5-HT-immunoreactive neurons occurs in the optic lobes as well as in the midbrain, and because the branching patterns of the individual PDHimmunoreactive neurons are not known, it cannot be discerned whether PDH- and 5-HT-immunoreactive neurons share a common pathway into the AMe and/or whether they share the same postsynaptic neurons in the AMe.

Some PDH-immunoreactive cells seem to have morphologies suitable for providing nonphotic input to the clock, namely input from the contralateral pacemaker. These $\mathrm{PDH}$-immunoreactive neurons with an unknown branching pattern in the optic lobe project through the posterior optic commissure into the contralateral optic lobe and might connect both accessory medullae directly. Current backfills and degeneration experiments combined with immunocytochemistry on the light and electronmicroscopic level indicate that there are projections from the contralateral optic lobe to the AMe and that $\mathrm{PDH}$-immunoreactive neurons form input as well as output synapses in the AMe (T. Reischig and M. Stengl, unpublished observations). Because both accessory medullae are the presumptive pacemaking centers in the cockroach (Homberg et al., 1991; Stengl and Homberg, 1994; Reischig and Stengl, 1996), PDH-immunoreactive neurons that anatomically connect both accessory medullae might couple both pacemakers. Previous cooling experiments indicated that both pacemakers are tightly coupled in cockroaches and that coolingdependent phase shifts of one pacemaker are transferred (apparently via neuronal connections) to the contralateral pacemaker (Page, 1981). The neuronal equivalent of this apparently direct coupling pathway and the mechanisms of coupling remained elusive. The possibility of a subpopulation of PDHimmunoreactive neurons being a neuronal substrate for the so far unknown coupling pathway is supported further by lesion experiments that revealed a correlation between the number of regenerating $\mathrm{PDH}$-immunoreactive commissures and changes in the period of the regained locomotor activity rhythm (Homberg and Stengl, 1994). The shortening of the period of the regained 
locomotor activity rhythm in animals with supernumerary regenerated $\mathrm{PDH}$-immunoreactive commissures (Stengl and Homberg, 1994) agrees with previous experiments showing that both pacemakers together generate a shorter period of the locomotor activity rhythm compared with a single pacemaker (Page, 1978).

The data presented in this study do not reveal whether PDHimmunoreactive neurons act only upstream of the pacemaker or whether they are also an integral part of the endogenous oscillator. Because in Drosophila melanogaster PDH-immunoreactive cells contain essential molecular components of the pacemaker, and they seem to be necessary for the expression of circadian locomotor rhythms, it might be possible that $\mathrm{PDH}$-immunoreactive cells are pacemaker neurons per se in insects (Helfrich-Förster and Homberg, 1993; Hall, 1995; Helfrich-Förster, 1995, 1997; HunterEnsor et al., 1996). Thus, it needs to be tested whether individual PDH-immunoreactive neurons are able to generate circadian membrane and action potential rhythms (R. Lösel, B. Petri, M. Stengl, and U. Homberg, unpublished observations), as has been shown for mollusk and vertebrate pacemaker cells (Michel et al., 1993; Welsh et al., 1995).

In conclusion, this study presents the first direct physiological evidence of the circadian function of the neuropeptide PDH. This as well as several other lines of evidence suggest that $\mathrm{PDH}$ immunoreactive neurons with their primary arborization area in the AMe are a nonphotic input into the clock and/or circadian pacemakers per se in the cockroach L. maderae.

\section{REFERENCES}

Chiba Y, Tomioka K (1987) Insect circadian activity with special reference to the localization of the pacemaker. Zool Sci 4:945-954.

Colwell CS, Page TL (1989) The electroretinogram of the cockroach Leucophaea maderae. Comp Biochem Physiol 92A:117-123.

Colwell CS, Page TL (1990) A circadian rhythm in neural activity can be recorded from the central nervous system of the cockroach. J Comp Physiol [A] 166:643-649.

Enright JT (1965) The search for rhythmicity in biological time-series. J Theor Biol 8:426-468.

Hall JC (1995) Tripping along the trail to the molecular mechanisms of biological clocks. Trends Neurosci 18:230-240.

Helfrich-Förster C (1995) The period clock gene is expressed in central nervous system neurons which also produce a neuropeptide that reveals the projections of circadian pacemaker cells within the brain of Drosophila melanogaster. Proc Natl Acad Sci USA 92:612-616.

Helfrich-Förster C (1997) Putative output pathways from Drosophila's circadian clock. J Neurogenet, in press.

Helfrich-Förster C, Homberg U (1993) Pigment-dispersing hormoneimmunoreactive neurons in the nervous system of wild-type Drosophila melanogaster and of several mutants with altered circadian rhythmicity. J Comp Neurol 337:177-190.

Homberg U, Würden S, Dircksen H, Rao KR (1991) Comparative anatomy of pigment-dispersing hormone-immunoreactive neurons in the brain of orthopteroid insects. Cell Tissue Res 266:343-357.

Hunter-Ensor M, Ousley A, Sehgal A (1996) Regulation of the Drosophila protein timeless suggests a mechanism for resetting the circadian clock by light. Cell 84:677-685.

Lösel R, Homberg U (1996) Anatomy and physiology of neurons in the area of the accessory medulla of the cockroach Leucophaea maderae. In: Göttingen neurobiology report (Elsner N, Schnitzler H-U, eds), p 27. Stuttgart, Germany: Thieme.

Meinertzhagen IA, Pyza E (1996) Daily rhythms in cells of the fly's optic lobe: taking time out of the circadian clock. Trends Neurosci 19:285-291.

Michel S, Geusz ME, Zaritsky JJ, Block GD (1993) Circadian rhythm in membrane conductance expressed in isolated neurons. Science 259:239-241.

Nässel DR, Shiga S, Wikstrand EM, Rao KR (1991) Pigment-dispersing hormone-immunoreactive neurons and their relation to serotonergic neurons in the blowfly and cockroach visual system. Cell Tissue Res 266:511-523.

Nishiitsutsuji-Uwo J, Pittendrigh CS (1968a) Central nervous system control of circadian rhythmicity in the cockroach. II. The pathway of light signals that entrain the rhythm. Z Vgl Physiol 58:1-13.

Nishiitsutsuji-Uwo J, Pittendrigh CS (1968b) Central nervous system control of circadian rhythmicity in the cockroach. III. The optic lobes, locus of the driving oscillation. Z Vgl Physiol 58:14-46.

Page TL (1978) Interaction between bilaterally paired components of the cockroach circadian system. J Comp Physiol 124:225-236.

Page TL (1981) Effects of localized low-temperature pulses on the cockroach circadian pacemaker. Am J Physiol 240:R144-R150.

Page TL (1982) Transplantation of the cockroach circadian pacemaker. Science 216:73-75.

Page TL (1983a) Regeneration of the optic tracts and circadian pacemaker activity in the cockroach Leucophaea maderae. J Comp Physiol 152:231-240.

Page TL (1983b) Effects of optic-tract regeneration on internal coupling in the circadian system of the cockroach. J Comp Physiol 153:353-363.

Page TL (1985) Clocks and circadian rhythms. In: Comprehensive insect physiology, biochemistry, and pharmacology. Vol 6, Nervous system: sensory (Kerkut GA, Gilbert LI, eds), pp 577-652. Oxford: Pergamon.

Page TL (1987) Serotonin phase-shifts the circadian rhythm of locomotor activity in the cockroach. J Biol Rhythms 2:23-34.

Page TL, Barrett KR (1989) Effects of light on circadian pacemaker development II. Responses to light. J Comp Physiol [A] 165:51-59.

Page TL, Caldarola PC, Pittendrigh CS (1977) Mutual entrainment of bilaterally distributed circadian pacemakers. Proc Natl Acad Sci USA 58:14-46.

Petri B, Stengl M, Würden S, Homberg U (1995) Immunocytochemical characterization of the accessory medulla in Leucophaea maderae. Cell Tissue Res 282:3-19.

Pyza E, Meinertzhagen IA (1995) Monopolar cell axons in the first optic neuropil of the housefly, Musca domestica L., undergo daily fluctuations in diameter that have a circadian basis. J Neurosci 15:407-418.

Pyza E, Meinertzhagen IA (1996) Neurotransmitters regulate rhythmic size changes amongst cells in the fly's optic lobe. J Comp Physiol [A] 178:33-45.

Rao RK, Riehm JP (1993) Pigment-dispersing hormones. Ann NY Acad Sci 680:78-88.

Reischig T, Stengl M (1996) Morphology and pigment-dispersing hormone immunocytochemistry of the accessory medulla, the presumptive circadian pacemaker of the cockroach Leucophaea maderae: a light- and electron-microscopic study. Cell Tissue Res 285:305-319.

Roberts SK (1965) Photoperception and entrainment of cockroach activity rhythms. Science 148:958-959.

Roberts SK (1974) Circadian rhythm in cockroaches. Effects of optic lobe lesions. J Comp Physiol 88:21-30.

Roenneberg T, Morse D (1993) Two circadian oscillators in one cell. Nature 362:362-364.

Sachs L (1969) Statistische Auswertungsmethoden. Berlin: Springer.

Sokolove PG (1975) Localization of the optic lobe circadian pacemaker with microlesions. Brain Res 87:13-21.

Sokolove PG, Bushell WN (1978) The chi square periodogram: its utility for analysis of circadian rhythms. J Theor Biol 72:131-160.

Stengl M (1995) Pigment-dispersing hormone-immunoreactive fibers persist in crickets which remain rhythmic after bilateral transection of the optic stalks. J Comp Physiol [A] 176:217-228.

Stengl M, Homberg U (1994) Pigment dispersing hormone-immunoreactive neurons in the cockroach Leucophaea maderae share properties with circadian pacemaker neurons. J Comp Physiol [A] 175:203-213.

Welsh DK, Logothetis DE, Meister M, Reppert SM (1995) Individual neurons dissociated from rat suprachiasmatic nucleus express independently phased circadian firing rhythms. Neuron 14:697-706.

Wiedenmann G (1977) Two activity peaks in the circadian rhythm of the cockroach Leucophaea maderae. J Interdiscip Cycle Res 8:378-383.

Wills SA, Page TL, Colwell CS (1985) Circadian rhythms in the electroretinogram of the cockroach. J Biol Rhythms 1:25-37.

Würden S, Homberg U (1994) Anatomy and physiology of neurons innervating the accessory medulla in the brain of the locust Schistocerca gregaria. In: Göttingen neurobiology report 1994 (Elsner N, Breer H, eds), p 463. Stuttgart, Germany: Thieme. 